\title{
Belphégor
}

\section{Faune diabolique et flore divine dans la légende fantastique espagnole «La azucena silvestre » (1845), de José de Zorrilla}

Justine Pédeflous

\section{OpenEdition}

Journals

Édition électronique

URL : http://journals.openedition.org/belphegor/445

DOI : 10.4000/belphegor.445

ISSN : 1499-7185

Éditeur

LPCM

Référence électronique

Justine Pédeflous, « Faune diabolique et flore divine dans la légende fantastique espagnole «La azucena silvestre » (1845), de José de Zorrilla », Belphégor [En ligne], 12-1 | 2014, mis en ligne le 15 juin 2014, consulté le 20 avril 2019. URL : http://journals.openedition.org/belphegor/445 ; DOI :

10.4000/belphegor.445

Ce document a été généré automatiquement le 20 avril 2019.

\section{(c) (1) $\Theta \Theta$}

Belphégor est mis à disposition selon les termes de la Licence Creative Commons Attribution - Pas d'Utilisation Commerciale - Pas de Modification 4.0 International. 


\title{
Faune diabolique et flore divine dans la légende fantastique espagnole \\ « La azucena silvestre » (1845), de José de Zorrilla
}

\author{
Justine Pédeflous
}

«Los peñascos

De Monserrate en su áspero fragor La luz esconden que sus rayos toma

En las pupilas del potente Dios. »

« Les rochers

De Montserrat dans leur fracassante rugosité Occultent la lumière qui prend sa source

Dans les pupilles de Dieu tout-puissant. »

(« La azucena silvestre »)

1 Au début d'une de ses légendes parue en 1840, l'écrivain espagnol José de Zorrilla estime que le genre fantastique pratiqué par Hoffmann est « inopportun en Espagne ${ }^{1}$ » en raison de la différence d'idiosyncrasie entre l'Allemagne, pays brumeux où règnent naturellement fantômes et lutins, et l'Espagne, dont le "soleil brillant donnerait aux contours de ses esprits terrifiants des couleurs chatoyantes ", annulant ainsi tout leur mystère (Zorrilla 1884 : 359). Il s'agit là d'une opinion courante à l'époque, aussi bien en Espagne qu'à l'étranger ${ }^{2}$. Cependant, Zorrilla est un des auteurs à avoir le plus contribué à l'essor du genre fantastique dans l'Espagne du XIX ${ }^{\mathrm{e}}$ siècle : le texte dans lequel il rejette le conte hoffmannien est pourtant sous-titré "cuento fantástico ». Sous cette apparente contradiction, apparaît néanmoins une poétique cohérente et originale du fantastique.

Dans le corpus légendaire de cet auteur, «La azucena silvestre » a la particularité de se dérouler dans un milieu naturel, la montagne de Montserrat en Catalogne, alors que les 
autres légendes ont un cadre urbain, l'auteur ayant une prédilection pour les églises, les monastères, et les ruelles éclairées par la lueur d'un crucifix. À la différence de ces légendes, où l'action divine opère généralement par l'intermédiaire d'un élément inorganique (une statue de la Vierge ou de Jésus-Christ), dans «La azucena silvestre », le fantastique est directement lié à la nature : la centralité de celle-ci est suggérée par le titre même de la narration, «Le lys sauvage ». Nous verrons comment l'auteur traite le paradoxe d'une « nature surnaturelle » qui, ni neutre ni homogène, est traversée par la cosmologie manichéenne propre au christianisme. L'étude de la nature fantastique dans "La azucena silvestre» sera également l'occasion de mettre en évidence la poétique problématique de la légende zorrillesque, entre tradition religieuse espagnole et fantastique moderne exogène. La dimension fantastique de l'œuvre de Zorrilla a déjà été étudiée de façon globale (Margherita Bernard 1995 ; Sebold 1995) mais sans que le monde naturel ne fasse l'objet d'une attention particulière dans son rapport avec la poétique fantastique.

\section{Zorrilla et le genre légendaire}

3 Entre 1837 et 1883 , José de Zorrilla $(1817-1893)^{3}$ publie une quarantaine de poèmes narratifs que l'on désigne généralement par le terme générique de «légende ». Zorrilla emploie indistinctement les termes «leyenda» et "tradición" pour se référer à des œuvres qui contiennent un élément surnaturel (à l'exception de «Honra y vida que se pierden no se cobran, mas se vengan, leyenda»). À l'inverse, ses œuvres « réalistes » sont qualifiées de "romances » (au sens espagnol de « romancero $^{4}$ » quoique Zorrilla ne respecte pas toujours la métrique du "romance $\left.e^{5} »\right)$ lorsque leur thème est historique et de " novelescas» quand elles ne mettent en scène aucun personnage historique (souvent il s'agit d'histoires d'amour).

4 Nous avons vu que l'auteur semblait rejeter la poétique fantastique d'Hoffmann, bien qu'il compose des « légendes » incluant des éléments surnaturels. Zorrilla oppose en effet deux types de « fantaisies » : d'un côté celles d'Hoffmann, qui, selon lui, « ne seront considérées en Espagne que comme les folies et les songes d'une imagination égarée "; de l'autre, un genre fantastique nouveau, la «fantaisie religieuse [...] [et] populaire» (Zorrilla 1884 : 359), qui ne répugne pas à l'Espagne, pays «religieux jusqu'au fanatisme ». Donnant l'exemple d'une de ses légendes, "Margarita la tornera. Tradición», dans laquelle le merveilleux chrétien a remplacé le surnaturel allemand, Zorrilla propose donc une poétique nouvelle du genre, paradoxalement en le reliant à la tradition espagnole.

5 Ainsi, dans la réédition de ses légendes en 1884, Zorrilla cite la source de «Margarita la tornera »: pour composer cette légende, il dit s'être inspiré de la littérature mariale espagnole (Cantigas de Santa María, d'Alphonse X, XIII ${ }^{e}$ siècle ; Milagros de Nuestra Señora, de Gonzalo de Berceo, XIII siècle), d'un moine italien du XIV ${ }^{\mathrm{e}}$ siècle et du père Raynaud, un jésuite français du XVII ${ }^{\mathrm{e}}$ siècle. D'autres corrélations peuvent être mises en évidence, avec un auteur du siècle d'or, Cristóbal Lozano.

6 «La azucena silvestre » est sans doute l'une des œuvres zorrillesques les plus influencées par la tradition espagnole. Elle revisite la vieille légende catalane de Juan Garín, présente dans les chroniques de Catalogne $\mathrm{du}^{\mathrm{xVI}}{ }^{\mathrm{e}}$ au $\mathrm{xVIII}^{\mathrm{e}}$ siècle $^{6}$, puis éditée de façon indépendante dans la "literatura de cordel », équivalent espagnol de la «Bibliothèque bleue », du $\mathrm{XVIII}^{\mathrm{e}}$ au $\mathrm{xIX}^{\mathrm{e}}$ siècle. La première œuvre " de cordel» a connu plusieurs 
rééditions, jusqu'en 1845 : La vida de fray Juan Garín, y de la penitencia, que hizo en la Montaña de Monserrate, y de la Fundación de la Casa, y del aparecimiento de la Virgen María, de Juan Pablo Font (1760). La seconde a été publiée pour la première fois en 1767 à Madrid par Hilario Santos Alonso, chez l'imprimeur Manuel José Martín, qui a été pris pour l'auteur de l'œuvre dans l'édition de 1778 : Historia verdadera de la aparición de nuestra Señora de Monserrate, $y$ los condes de Barcelona, con los sucesos extraños, y maravillosos de la infanta Doña Riquilda, y el Ermitaño Fr. Juan Guarín. Sacada de muchos, y graves autores, como el Doctor Serra, Pujadas, Diago, Doménech, y otros Historiadores del Principado de Cataluña ${ }^{7}$. On trouve une dernière version «de cordel » de la légende: Historia de la aparición de Nuestra Señora de Montserrat, con los extraños y maravillosos sucesos del anacoreta Fr. Juan Garin, publiée anonymement à Madrid chez Marés entre 1865 et 1880 . Cette version a également été éditée avec le même titre, à Madrid chez Hernando, sans date ni nom d'auteur.

7 La légende a inspiré de nombreuses œuvres littéraires : dès 1588, elle donne lieu à un poème épique, El Monserrate, de Cristóbal Virués, bien qu'elle ne soit pas l'unique thème de l'œuvre. La légende a aussi inspiré une pièce de théâtre, publiée sans date ni nom d'auteur, Comedia nueva. El monstruo de Cataluña y peñas de Monserrate. Fray Juan Guarín, à Barcelone, chez Juan Francisco Piferrer. Les dates d'activité de cet imprimeur (1798-1846) suggèrent que l'œuvre a été composée au début du XIX ${ }^{e}$ siècle, c'est-à-dire après les deux versions « de cordel » et vraisemblablement avant « La azucena silvestre ». C'est à partir de l'époque romantique que la légende intéresse le plus la littérature ${ }^{8}$. Zorrilla est le premier à en proposer une réécriture et nous verrons que, s'il conserve la plupart de ses composantes, «La azucena silvestre » constitue néanmoins une version originale et personnelle de la légende.

\section{« La azucena silvestre » et la tradition religieuse espagnole : la nature diabolique}

8 L'écrivain espagnol reprend les divers épisodes de la légende catalane : la tentation de l'ermite Juan Guarino ${ }^{9}$ par le diable par l'intermédiaire indirect de la fille du comte de Barcelone venue se faire soigner par lui, et d'un faux ermite (le diable déguisé) qui le pousse au crime ; le viol puis le meurtre de la jeune fille; la pénitence donnée par le Pape à Guarino de marcher à quatre pattes sans regarder le ciel ; la capture de Guarino devenu monstrueux par le comte de Barcelone ; le pardon divin par l'intermédiaire d'un enfant et le pardon du comte ; la découverte du corps de la jeune fille encore en vie ; l'apparition de la Vierge Marie. On remarque que le surnaturel est présent du début à la fin du récit, sous la forme du merveilleux chrétien. Selon la cosmovision médiévale ainsi que pour le chrétien croyant jusqu'au XIX ${ }^{e}$ siècle, l'existence du diable, de même que de Dieu et de la Vierge, était aussi réelle que le monde sensible et n'avait pas besoin d'être justifiée. Cependant, leur apparition sur Terre sous une forme palpable était considérée comme une possibilité, mais sa rareté, rompant avec la réalité quotidienne, la rendait improbable. Cela explique que même dans la littérature religieuse médiévale, et malgré une vision du monde moins soumise aux lois naturelles, les personnages témoins d'une apparition divine ou diabolique, ou d'un miracle, aient comme première réaction de la surprise, voire de la terreur, de même que les personnages de la littérature fantastique face à un phénomène surnaturel non religieux. La différence entre le "merveilleux chrétien " (ainsi nommé bien que son décor soit un monde semblable à l'univers du lecteur, et non pas un monde aux coordonnées totalement différentes), et le fantastique, 
réside non pas dans la réaction de surprise des personnages, qui serait absente dans la première, présente dans la seconde, mais dans l'interprétation du phénomène une fois passé l'étonnement. Si le croyant replace l'apparition ou le miracle dans sa cosmovision religieuse, lui donnant ainsi une raison d'être et lui ôtant sa dimension terrifiante, le personnage de la littérature fantastique n'a pas cette possibilité, du moins pas immédiatement : soit il n'y a aucune explication plausible à l'événement, soit il y en a plusieurs qui se chevauchent sans qu'il y en ait aucune de pleinement satisfaisante.

La légende de Juan Guarino appartient en propre au merveilleux chrétien. En effet, dans plusieurs versions, les interventions divine et diabolique provoquent dans un premier temps de la stupeur chez les témoins de la scène. Lorsque la surprise des spectateurs n'est pas explicitement exprimée, le narrateur utilise néanmoins un vocabulaire tout à fait significatif de la rupture de la quotidienneté : "merveilles", "prodige très étrange et singulier » (Santos Alonso 23). Par la suite, l'origine divine du phénomène est reconnue et acceptée, ce qui fait basculer le texte dans le «merveilleux» selon la définition todorovienne :

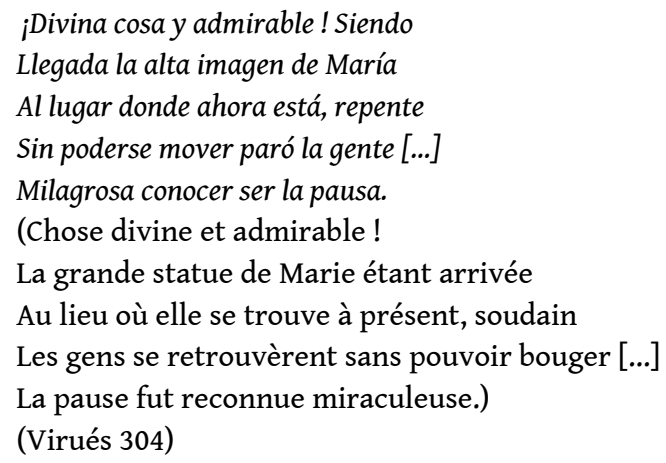

En cela, Zorrilla suit donc la tradition dont il s'inspire en mettant en scène la stupeur des personnages, par exemple en entendant l'enfant et la bête parler, après quoi, le Comte attribue à Dieu le prodige auquel il vient d'assister :

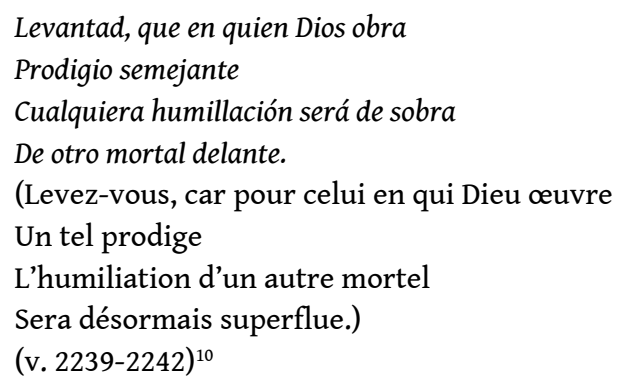

11 Zorrilla est aussi fidèle à ses sources concernant l'étrange pénitence de l'ermite et ses conséquences sur son être. En effet, les différentes versions de la légende décrivent l'animalisation de Guarino due à de longues années passées à quatre pattes :

De un vello espeso y largo cobijada

Con gran monstrosidad la piel tenía,

Que revuelto, encrespado, y descompuesto

Hacía fiero el cuerpo, y bravo el gesto.

[La bête] avait la peau monstrueusement

Recouverte de poils épais et longs,

Qui, hérissés, en bataille et en désordre,

Rendait son corps sauvage, et son attitude féroce.

(Virués 279) 
Dans la pièce de théâtre, une didascalie indique : « Va saliendo Juan Garín, andando como cuadrúpedo, con manos y rodillas, vestido con botarga de oso, el pelo tendido sobre el rostro, y barba muy prolija negra.» («Juan Garín sort, en marchant comme un quadrupède, sur ses mains et ses genoux, vêtu d'une peau d'ours, ses cheveux retombant sur son visage, et avec une barbe noire très abondante. ») Juan Pablo Font ajoute qu'il mange de l'herbe comme les animaux : ce détail provient de la légende de Saint Jacques, selon Miquel y Planas. Si Zorrilla ne conserve pas le détail de l'herbe, il est fidèle à la tradition concernant la description de la bête, y ajoutant simplement un développement moderne à propos de sa race problématique : «Del jerbo tenía el paso, / del mono la formación.» («Il avait la démarche de la gerboise, / et l'apparence du singe.») (v. 1878-1879) Loin d'être uniquement un prodige supplémentaire, ou un simple procédé narratif visant à rendre vraisemblable la rencontre entre Garín et le comte, l'étrange métamorphose de l'ermite, dont l'importance est signalée par la gravure dans une des versions antérieures ${ }^{11}$, a une signification symbolique. Si elle est justifiée par des causes naturelles dans certaines versions ${ }^{12}$, dans d'autres, une explication plus transcendante apparaît, celle du châtiment divin. Dans Historia verdadera, c'est le Pape, représentant de Dieu sur Terre, qui explicite le lien de causalité entre la bestialité et la notion de châtiment : « le puso por penitencia, que nunca mirase al Cielo, a quien había ofendido ; y pues como bruto animal se había dejado llevar de su sensualidad, y torpeza, que anduviese con las manos por la tierra como bestia.» ( «Il lui imposa comme pénitence, de ne jamais regarder le Ciel, qu'il avait offensé ; et, comme il s'était laissé entraîner par sa sensualité et sa turpitude comme un animal, de marcher les mains par terre comme une bête. ») (Santos Alonso 18)

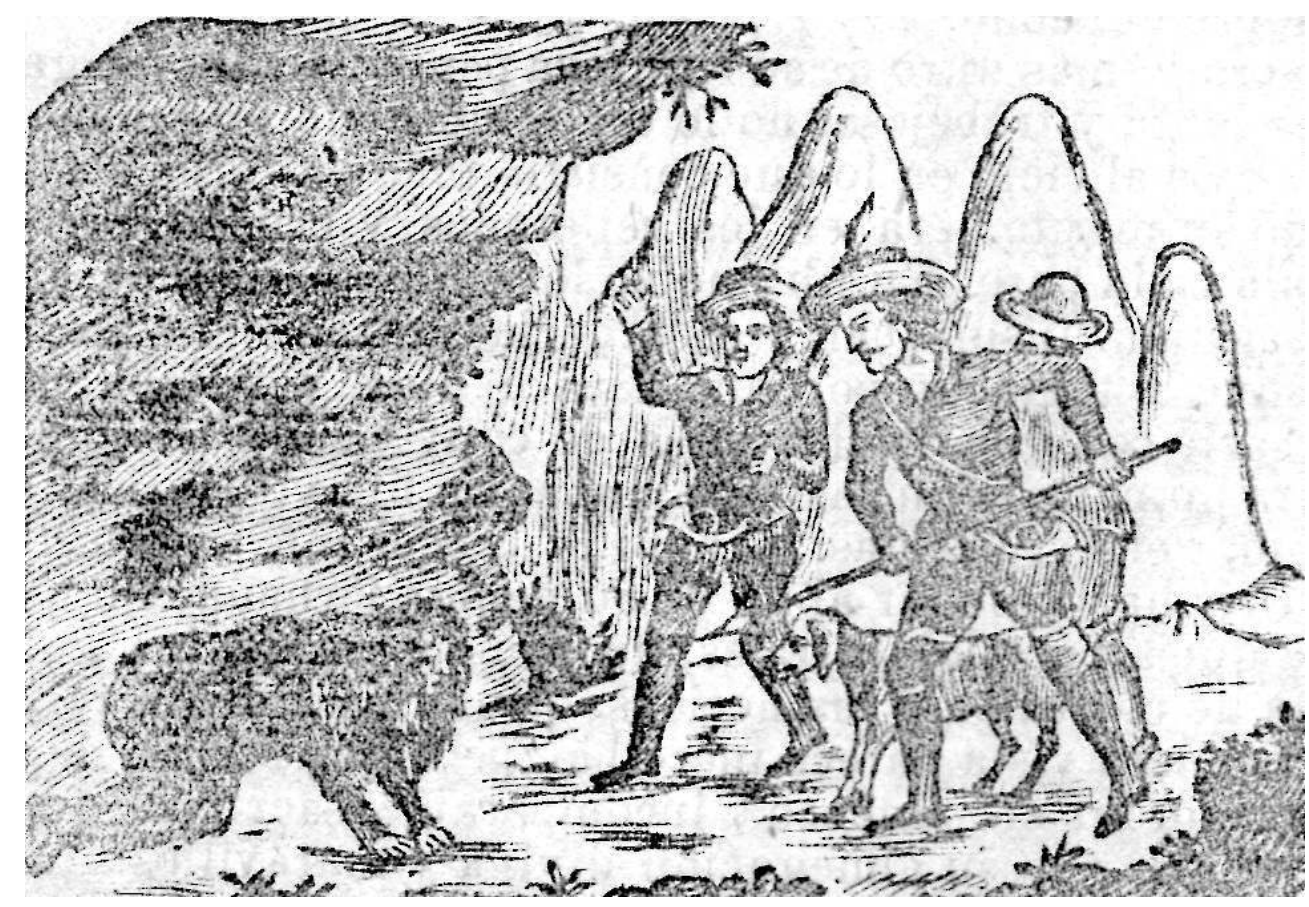

Garin transformé en animal

DR

13 Ainsi, depuis l'interprétation paulinienne puis augustinienne des Écritures, et surtout à partir du Moyen Âge, le corps est diabolisé par la religion chrétienne et la sensualité devient synonyme de bestialité. Guarino doit vivre comme une bête pour faire pénitence 
mais le merveilleux chrétien transforme la comparaison en réalité. Il s'agit là d'un prodige répondant à une logique analogique courante dans la littérature religieuse. Ainsi, dans un romance de 1858, une jeune débauchée est transformée par Dieu en mule en raison de la symbolique de cet animal ${ }^{13}$. Dans la littérature populaire, l'animal est souvent associé au châtiment divin. Dans Fructus Sanctorum, Alonso de Villegas rapporte l'histoire d'une pècheresse qui apparaît à deux saints hommes en rêve et leur raconte sa pénitence : pour chaque péché commis par un de ses membres, le membre en question est tourmenté par un animal ${ }^{14}$. Selon la contradiction propre à la littérature religieuse populaire, l'animal, s'il sert d'opérateur de la volonté divine, est aussi associé au démon, qui apparaît lui-même comme un adjuvant de Dieu : dans un romance, un noble ayant fourni un faux témoignage contre une jeune fille qu'il avait tenté de séduire se fait arracher la langue et emporter aux enfers par des chiens mais l'épisode est interprété comme une action divine 15. Conformément à la pensée médiévale, la nature n'est donc pas considérée comme un ensemble régi par des lois observables scientifiquement mais comme la création de Dieu ${ }^{16}$ : les phénomènes naturels sont donc interprétés suivant une grille allégorique renvoyant à la cosmovision chrétienne. L'animalisation de l'ermite s'explique donc par l'association des notions de bestialité et de démoniaque alors que la ré-humanisation de Guarino intervient au moment du pardon divin : redevenu par sa pénitence une créature de Dieu, il peut donc reprendre forme humaine.

Cette vision diabolique de la faune est renforcée chez Zorrilla par la description de la montagne catalane. Si celle-ci était décrite dans le poème de Virués comme un paradis terrestre, dans "La azucena silvestre », elle répond plutôt au topos du désert érémitique, aussi bien en raison de la dureté de ses conditions de vie, qui favorise la pénitence, que de sa connotation diabolique (Gutiérrez Martínez 758). En effet, Zorrilla, en plaçant dans d'« obscures cavernes » des reptiles et des bêtes sauvages, annonce grâce aux premiers la future présence du diable ${ }^{17}$, et par les secondes, la future transformation de Guarino. À la première lecture, même si la symbolique de ces éléments n'est pas décodée, ceux-ci contribuent à façonner un décor inquiétant. Par la suite, suivant la tradition médiévale, l'écrivain met en scène le diable sous une forme animale : il est décrit comme ayant d'« immenses ailes » (v. 1250). À l'inverse, les versions précédentes ne donnaient aucune description du diable, le désignant simplement comme tel. Si dans son association de l'animal et du démoniaque, Zorrilla suit les versions antérieures de la légende ainsi que la tradition médiévale, l'ajout de certains éléments absents des premières laisse penser que « La azucena silvestre » constitue une réécriture personnelle de la légende.

\section{Une légende romantique et fantastique : la nature divine}

15 La figure du diable illustre la poétique de Zorrilla, entre tradition et rénovation. En effet, si le démon correspond à son image médiévale, il a également une caractéristique qui le rapproche plutôt de la littérature romantique. Le poète insiste sur la notion de taille (" gigantesque figure », v. 1249), ce qui évoque la fascination gothique et romantique pour le diable, figure ambivalente à la fois majestueuse et terrifiante, notamment représentée par Lewis dans Le Moine (1796) et par Byron dans Caïn (1822).

On observe également une influence du romantisme sur Zorrilla dans le traitement de la figure féminine. En effet, dans certaines autres versions de la légende, la fille du comte 
n'était même pas nommée. Dans $E l$ Monserrate, elle est même rendue responsable de la tentation de Guarino : si elle vient voir l'ermite car elle est possédée par le diable comme dans les versions ultérieures, par la suite, après que Guarino l'a exorcisée, il est suggéré qu'elle le séduit ${ }^{18}$. Cette conception misogyne de la femme, conforme à la tradition chrétienne, sera reprise de façon plus explicite encore dans la réécriture publiée par Marés et Hernando' ${ }^{19}$. À l'inverse, Zorrilla s'inspire plutôt des autres versions, dans lesquelles la jeune fille n'est qu'un pion inconscient du diable et insiste sur sa dimension de victime innocente. En effet, alors que les autres versions ne faisaient état que de sa grande beauté, le poète décrit longuement la jeune María dès le début du poème, en mettant l'accent sur sa pureté et son innocence :

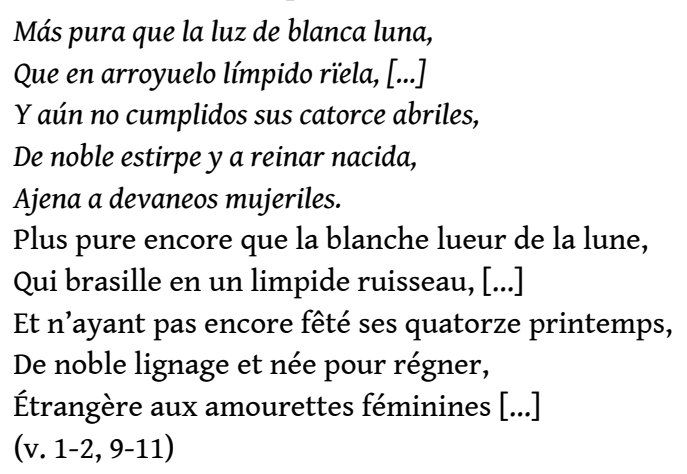

17 On retrouve dans cette description la femme romantique par excellence, virginale, éthérée. La pureté de la jeune fille est également exprimée par son prénom: le plus souvent appelée Riquilda, parfois María, la jeune fille reçoit ici le prénom de la Vierge, afin de montrer leur ressemblance et de préfigurer la fin du poème, le sauvetage d'une María par une autre. C'est pourquoi elle est décrite comme la victime innocente de Guarino et non pas comme sa tentatrice : "víctima de mi torpe desvarío / su virginal pureza sucumbió.» («victime de ma folle concupiscence / sa virginale pureté succomba.») (v. 2344-236)

Or, il convient de remarquer que dès le début, María est associée au monde floral au moyen d'une métaphore, qui exprime à nouveau la dimension virginale du personnage en même temps qu'elle annonce la fin du poème :

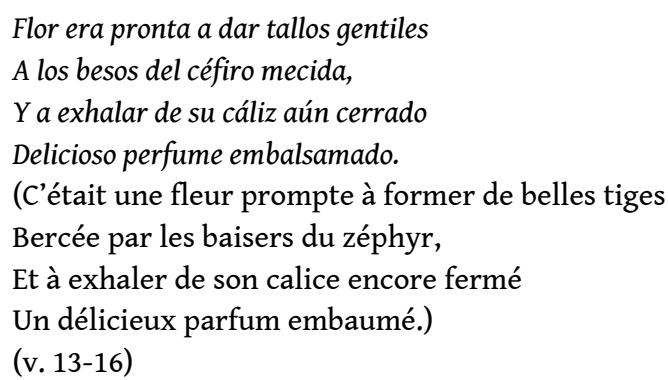

9 Zorrilla poétise substantiellement le dénouement de la légende. Dans toutes les versions, la jeune fille est retrouvée vivante dans la tombe creusée par Guarino avec comme seule marque résiduelle du crime, une trace écarlate, à l'endroit où l'ermite l'a égorgée. Dans " La azucena silvestre", María ne présente pas une trace mais une blessure fraîche lorsqu'elle est trouvée par l'ermite et son père, et c'est de cette blessure que se nourrit le lys qui surplombe sa tombe. Quand les deux hommes la trouvent ainsi, ils craignent de tuer la jeune fille, qui semble endormie, s'ils arrachent la fleur, comme si une mystérieuse connexion existait entre les deux. Cependant, n'y tenant plus, le père finit par l'arracher, et c'est à ce moment-là que María revient à la vie et que la Vierge apparaît pour prendre 
dans ses mains la fleur miraculeuse. Ce miracle est préparé par une description détaillée de la vallée qui tranche avec la description de la montagne du début du poème: il ne s'agit pas du désert rocailleux où se cachent reptiles et bêtes sauvages mais d'un véritable locus amoenus traversé par une rivière, jonché de fleurs diverses, et peuplé de lapins et de papillons ${ }^{20}$. Le poète insiste sur la dimension colorée et joyeuse du printemps, mais surtout sur la renaissance qu'il suppose, préfigurant ainsi la résurrection de la jeune fille : «Todo en ella juventud y vida, / todo en ella armonía, luz y aroma.» ("Tout en elle était jeunesse et vie, / tout en elle était harmonie, lumière et parfum. ») (v. 2680-1) Par la suite on retrouve les caractéristiques de la montagne aride et escarpée mais un élément de la vallée, une source, est venu se greffer sur cet univers hostile et "stérile», le rendant vivant et fécond. C'est précisément dans cet oasis miniature, qu'a poussé la «purísima azucena " («lys très pur »), "fresca, olorosa, amena » («frais, parfumé, amène ») (v. 2762-2763). Le poète entoure cette fleur d'une aura de mystère en lui attribuant une vie étrangère à sa nature : «[...] dándola oculto movimiento y vida, / hacían de la cándida azucena / un animado ser. » («[...] lui donnant un mouvement et une vie cachés, /transformaient le lys blanc / en un être animé.») (v. 2774-2776) En outre, il annonce l'étrange connexion entre la fleur et la jeune fille en réutilisant dans la description de la première des termes qu'il avait employés pour décrire la seconde : «su tallo verde, fresco, alto flexible, / mecido por el aura. » (" sa tige verte, fraîche, grande, flexible, / bercé par le zéphir. ») (v. 2271-2772) En tissant un réseau de correspondances poétiques, il prépare le lecteur à un phénomène surnaturel lié à la figure de la métaphore. En effet, l'association imagée fleur / jeune fille devient une réalité tangible dans leur alliance vitale, se nourrissant l'une de l'autre. Cependant, cette connexion n'est pas double mais triple : le prodige n'est pas naturel mais divin. La Vierge Marie, déjà associée à la jeune fille par son nom, est aussi liée dans le texte au lys sauvage au moyen de l'adjectif superlatif «purísimo», qui désigne également 
la fille du comte ${ }^{21}$ : le triangle est complété lorsque la Vierge prend le lys et rend la vie à María.

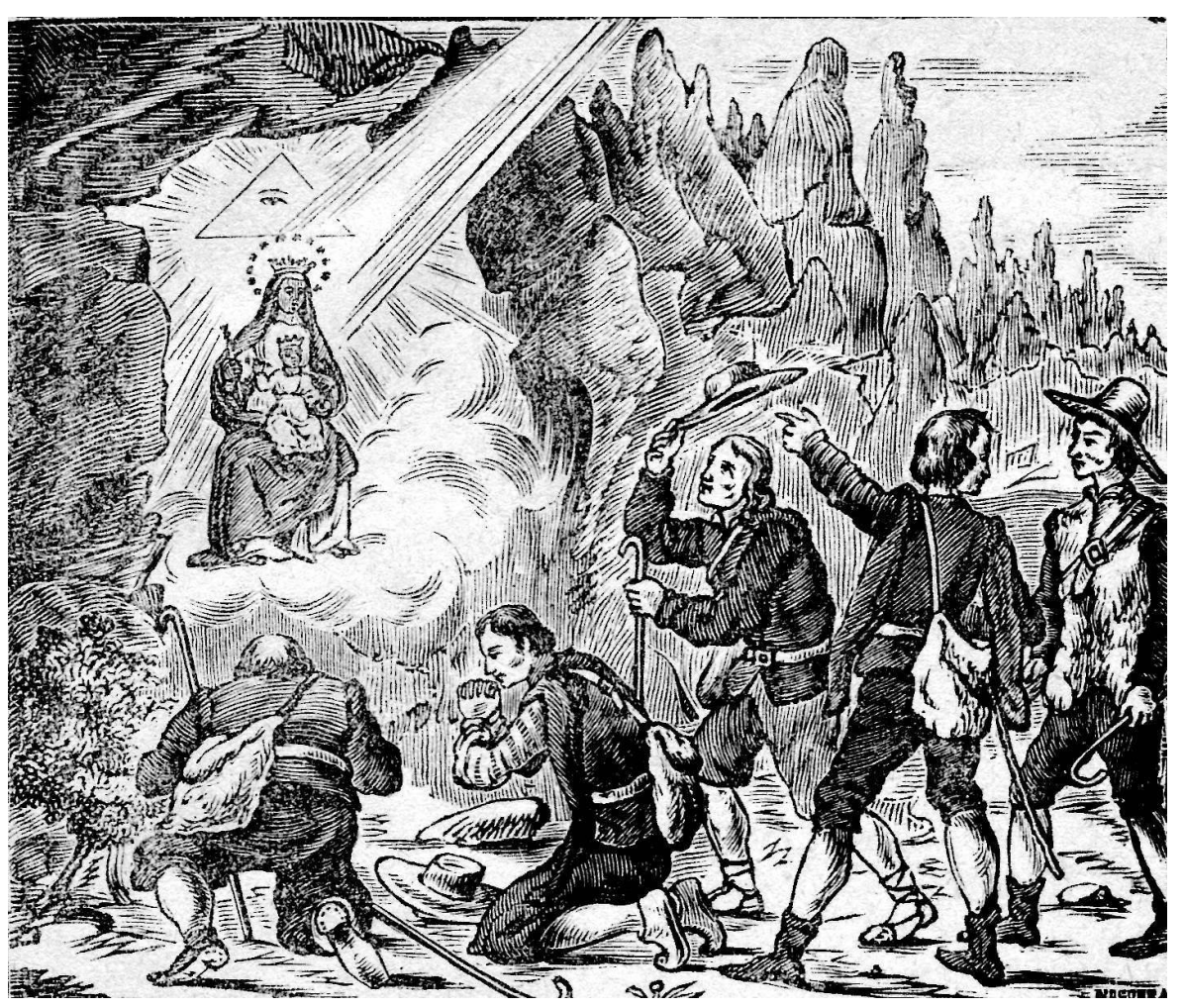

Apparition de la Vierge aux bergers

DR

Cette fin poétique et apothéotique a pu être inspirée à Zorrilla par l'illustration de l'ouvrage de Serra y Postius, qui représente la Vierge au milieu de la montagne, une fleur à la main : cette illustration a aussi inspiré la gravure de l'Historia verdadera du XVIII siècle. Malgré ces deux illustrations, aucun texte ne fait allusion à une quelconque fleur : il s'agit donc de l'ajout majeur de Zorrilla. On pourrait penser que cet intérêt pour la nature provient du romantisme ${ }^{22}$ : cependant, en Espagne, la nature est rarement prise comme sujet de poèmes romantiques, afin d'évoquer la corrélation entre l'état d'esprit du poète et le monde extérieur (Allison Peers 412). Ainsi, dans « La azucena silvestre », le poète indique même que le caractère riant de la vallée est en contradiction ouverte avec les sentiments sombres de l'ermite et du comte. Le monde naturel n'a pas ici pour fonction de représenter les émotions d'un spectateur mais au contraire de révéler l'action divine sur Terre. Nous avons vu que l'animalité était liée au démoniaque bien que ce soit Dieu qui permette la ré-humanisation de Guarino. À la fin du poème, l'action de la Vierge, qui, dans les autres versions, était directe mais n'était pas représentée (c'est la jeune fille qui raconte que c'est la Vierge qui l'a maintenue en vie), est ici médiatisée et symbolisée par la fleur. En cela, Zorrilla est proche de la représentation médiévale de la divinité (ou du diable) qui, pour être intelligible, était associée à un élément tangible : dans les miracles de Berceo, on trouve des comparaisons de la Vierge au pain, afin de la rendre plus proches des classes populaires. De façon plus précise, le motif de la fleur divine a pu lui être inspiré par le miracle III de Berceo «El clérigo y la flor»: un moine, fervent admirateur de la Vierge, est assassiné par des voleurs qui voient apparaître Marie leur ordonnant de l'enterrer dans le cimetière; quelques jours plus tard, ils le trouvent dans 
sa tombe avec une fleur lui sortant de la bouche et décident de l'enterrer dans le cimetière pour que son âme repose en paix. On voit que Zorrilla a considérablement augmenté et poétisé le miracle médiéval.

21 Il avait déjà utilisé la potentialité symbolique et poétique du motif de la fleur dans une autre légende, «La pasionaria ». À la fin, Clotilde, malade, demande à son amant Félix de couper la fleur qui avait poussé sous leur fenêtre, pour qu'elle meure avec elle. Malgré ses scrupules, considérant qu'il existe un mystérieux lien entre sa femme et la fleur, il la coupe et voit apparaître Aurora, son premier amour défunt, qui lui explique que son destin était lié à celui de Clotilde. On retrouve dans cette légende une description semblable de la fleur, «pura, olorosa, fresca / [...] que a los besos del aura se mecía » ("pure, parfumée, fraîche / [...] qui était bercée par les baisers du zéphir ») (Zorrilla 1884 : 377), et la même relation triangulaire que dans « La azucena silvestre ». Cependant, si cette légende n'est pas dénuée de spiritualité - la fleur de la passion pouvant être lue comme symbole de la passion, au sens étymologique du terme, quoique cette interprétation ne soit pas explicitée dans le texte - la passionnaire n'en reste pas moins une version profane du lys divin. Néanmoins, il est intéressant de constater l'utilisation d'un même motif comme levier surnaturel dans un poème sous-titré « légende religieuse » et dans un autre soustitré « conte fantastique ».

En effet, Zorrilla ne se contente pas de poétiser la légende médiévale, il y applique également une nouvelle poétique, celle du fantastique. On pouvait déjà trouver dans $\mathrm{El}$ Monserrate des éléments fantastiques, comme le suggère dans le prologue Baltasar de Escobar, lorsqu'il évoque la compatibilité à laquelle est arrivé Virués entre le «vraisemblable» et le «merveilleux »". Ainsi, les visions diaboliques sèment le doute chez Guarino, tout comme dans El monstruo de Cataluña. Cependant, dans la majorité des cas, les apparitions diaboliques et les miracles divins sont traités par les narrateurs sur le mode de l'affirmation, de la même façon que les épisodes réalistes.

Cela s'explique par le fait que les versions antérieures à «La azucena silvestre » ont une finalité autre que celle d'étonner. Dans les versions contenues dans des chroniques catalanes ainsi que dans Historia verdadera, le but affiché est d'expliquer l'origine du monastère de Montserrat : l'apparition de la Vierge à des bergers (voir la fig. 1) aurait conduit à la construction d'une chapelle, qui se serait par la suite transformée en monastère sous l'impulsion de la fille du comte ressuscitée, aidée d'un Guarino absous. Il s'agit donc ici d'une légende dite "étiologique ", qui entend expliquer l'origine d'un monument ou d'un lieu (Balzamo 2008). Néanmoins, toutes les versions ne poursuivent pas le même objectif, ou du moins pas exclusivement: El Monserrate insiste beaucoup plus sur la pénitence de Guarino, et sur sa longue et périlleuse pérégrination jusqu'à Rome, ce qui permet à l'auteur d'inclure des passages épiques sans rapport avec la légende originelle. En outre, si les autres versions soulignent l'omnipotence divine, Virués y associe de fréquentes imprécations contre le monde, dans la lignée de l'Ecclésiaste et en lien avec le «desengaño » du Siècle d'or. La pièce de théâtre insiste sur le pouvoir du diable sur l'humanité comme maître de l'illusion mais prouve la supériorité divine dans la capacité d'émerveillement : le miracle, démonstration frappante de la présence divine, a ici pour but de renouveler la foi.

24 Zorrilla va plus loin dans cette voie : en réunissant les deux miracles (la résurrection de María et l'apparition de la Vierge), normalement distincts, il accroît le caractère dramatique de la légende tout en s'éloignant de sa motivation étiologique. En revanche, il ajoute un élément quasi absent des versions antérieures, le mystère. Au lieu d'une 
narration linéaire et totalement explicite, il choisit de laisser des zones d'ombre grâce à la juxtaposition de scènes jonchées de détails apparemment insignifiants mais qui demandent la participation du lecteur afin de construire le sens du texte. Par exemple, alors que les autres versions identifiaient d'emblée le monstre poilu à Guarino et donnaient la pénitence comme cause de cette transformation, dans " La azucena silvestre ", la première partie se clôt sur le désespoir de l'ermite et sur celui du père ne trouvant plus sa fille, et la seconde s'ouvre sur la découverte d'une mystérieuse bête. La description de celle-ci, comprenant des traits humains, laisse entrevoir qu'il s'agit de l'ermite mais il faut attendre le miracle de l'enfant pour sortir de l'incertitude et la narration de Guarino pour connaître la cause de la métamorphose. De même, l'épisode de l'entrevue entre le comte et sa fille et un vieil homme qui leur enjoint de se rendre à Montserrat est empreint de mystère : si le vieillard possède un air de sainteté et s'envole avec le vent, ce qui suggère aux deux témoins qu'il s'agit d'un envoyé du ciel, d'autres éléments, des sensations vagues, contredisent cette interprétation, ce qui contribue à créer l'ambiguïté propre au fantastique. Le lecteur devra attendre le discours du diable à Guarino pour comprendre que l'aveuglement de María n'était pas dû à Dieu mais à lui, et que le vieillard n'était pas un envoyé divin mais diabolique.

Il y a également fusion entre le merveilleux chrétien et le fantastique moderne dans la corrélation déjà évoquée entre métaphore et réalité car elle permet de créer un réseau de correspondances analogiques qui témoignent d'un fonctionnement différent du système positiviste qui commence à se mettre en place au milieu du XIX ${ }^{e}$ siècle. Zorrilla s'éloigne de la tradition dans la mesure où le merveilleux chrétien est doublé d'éléments fantastiques qui indiquent la nostalgie romantique d'un passé poétique, religieux et merveilleux, par opposition à un présent prosaïque, sécularisé et dénué de toute magie.

Cette vision du monde romantique sera accentuée par le post-romantique Gustavo Bécquer (1836-1870) dans ses légendes en prose publiées entre 1858 et 1864 dans la presse. S'inspirant lui aussi de légendes traditionnelles espagnoles, il les recompose en fonction de ses idées artistiques et de sa cosmovision romantique. Par deux fois, il utilisera le motif de la fleur liée à une jeune fille : dans «La promesa » (1863), des fleurs poussent sur la tombe d'une jeune fille séduite puis abandonnée par un comte, et assassinée par ses frères. Dans «La rosa de pasión " (1864), qui s'inspire plutôt de «La pasionaria ", une jeune Juive amoureuse d'un Chrétien et convertie au christianisme subit à la place de son amant la fureur des Juifs qui avaient recréé pour lui tous les éléments de la Passion (croix, couronne d'épines). Sur la tombe de la jeune fille pousse une fleur inconnue en forme de couronne que l'on appelle "rose de la passion». Chez Bécquer, dont la foi est moins optimiste que celle de Zorrilla, la fleur n'est plus le signe de l'action divine mais l'indice remarquable d'une injustice ne devant pas être oubliée. De plus, elle intervient seulement à la fin du récit et n'en constitue pas un élément central comme c'était le cas chez Zorrilla, la «purísima flor » cristallisant à la fois le mystère et la poésie de la religion. On trouve chez Bécquer la même diabolisation de la bestialitée ${ }^{24}$ bien que la nature ne soit pas traversée par une ligne de fracture aussi marquée ${ }^{25}$ que dans « La azucena silvestre »: la nature devient en effet chez Bécquer un élément constitutif de la poétique fantastique de l'ambiguïé, que le poète romantique avait ébauchée et que l'écrivain post-romantique mène à son comble dans ses légendes. 


\section{BIBLIOGRAPHIE}

Anonyme. Comedia nueva. El monstruo de Cataluña y peñas de Monserrate. Fray Juan Guarín. Barcelone : Juan Francisco Piferrer, sans date.

Anonyme., Historia de la aparición de Nuestra Señora de Montserrat, con los extraños y maravillosos sucesos del anacoreta Fr. Juan Garin. Madrid : Marés, 1865-1880.

Anonyme. Historia de la aparición de Nuestra Señora de Montserrat, con los extraños y maravillosos sucesos del anacoreta Fr. Juan Garin. Madrid : Hernando, sans date.

Allison Peers, E. Historia del movimiento romántico español, tome I. Madrid : Gredos, $1973\left(2^{\mathrm{e}}\right.$ édition).

Balaguer, Víctor. Monserrate. Su historia. Sus tradiciones. Sus alrededores. Barcelone : A. Brusi, 1850.

Balzamo, Elena. « Le conte étiologique. » Fabula 49 (2008) : 87-105.

Bécquer, Gustavo. Leyendas. Madrid : Cátedra, 2006 [1858-1864].

Berceo, Gonzalo de. Milagros de Nuestra Señora. Madrid : Espasa-Calpe, 1991 [XIII ${ }^{\mathrm{e}}$ siècle].

Bernard, Margherita. « Dimensión fantástica y maravillosa en Zorrilla. » Actas del Congreso sobre José Zorrilla : una nueva lectura, Valladolid, 18-21 de octubre de 1993. Valladolid : Universidad Fundación Jorge Guillén, 1995. 241-250.

Bessière, Irène. Le récit fantastique : la poétique de l'incertain. Nancy : Berger-Levrault, 1973.

Entrambasaguas y Peña, Joaquín. « El Doctor Don Cristóbal Lozano. » Revista de archivos, bibliotecas y museos XLVIII, 7 - 9 (juillet-septembre 1928) : 209-231.

Font, Juan Pablo. La vida de fray Juan Garín, y de la penitencia, que hizo en la Montaña de Monserrate, y de la Fundación de la Casa, y del aparecimiento de la Virgen María. Barcelone : Juan Forns, 1760.

Guadalajara Medina, José. « El retrato del Anticristo en los textos castellanos medievales. » Actas del VI Congreso Internacional de la Asociación Hispánica de literatura medieval, tome I. Ed. José Manuel Lucía Megías. Alcalá : Universidad de Alcalá, 1997 : 729-736.

Gutiérrez Martínez, María del Mar. « Espacios y momentos peligrosos para el hombre medieval (Aparición del maligno en algunos textos del XIII al XIV). » Actas del VI Congreso Internacional de la Asociación Hispánica de literatura medieval, tome I. Ed. José Manuel Lucía Megías. Alcalá :

Universidad de Alcalá, 1997 : 757-768.

Juretschke, Hans. « La recepción de la cultura y ciencia alemana en España durante la época romántica ». Separata de Estudios románticos. Valladolid : Casa-Museo de Zorrilla, 1975 : 63-120.

Lavergne, Léonce de. « Mouvement littéraire de l'Espagne. Zorrilla. » Revue des Deux Mondes II (15 avril 1843) : 173-208.

Miquel y Planas, Ramón. La leyenda de Fray Juan Garín, ermitaño de Montserrat : Estudio sobre sus orígenes y formación. Barcelone : Orbis ,1940.

Santos Alonso, Hilario. Historia verdadera de la aparición de nuestra Señora de Monserrate, y los condes de Barcelona, con los sucesos extraños, y maravillosos de la infanta Doña Riquilda, y el Ermitaño Fr. Juan 
Guarín. Sacada de muchos, y graves autores, como el Doctor Serra, Pujadas, Diago, Doménech, y otros Historiadores del Principado de Cataluña. Cordoue : Juan Rodríguez de la Torre, sans date.

http://books.google.fr/books?id=48lYERh7TYwC\&printsec=frontcover\&dq=historia+verdadera + de+la

+aparicion\&source=bl\&ots=PWFf8SXr3b\&sig=EJlR9vPyOR97TR8yTOVJE9cRe7Y\&hl=fr\&sa=X\&ei=TAclUKHWAcq_0QXL2YCwCg\&ved=0CDg \%20verdadera\%20de\%20la\%20aparicion\&f =false

Pastoureau, Michel. Bestiaires du Moyen âge. Paris : Seuil, 2011.

Sebold, Russell. « Zorrilla en sus leyendas fantásticas a lo divino ». Actas del Congreso sobre José Zorrilla : una nueva lectura, Valladolid, 18-21 de octubre de 1993. Valladolid : Universidad Fundación Jorge Guillén, 1995 : 203-218.

Todorov, Tzvetan. Introduction à la littérature fantastique. Paris : Seuil, 1970.

Villegas, Alonso. Fructus Sanctorum, y quinta parte de Flos Sanctorum : que es libro de exemplos, así de hombres ilustres en santidad, como de otros cuyos hechos fueron dignos de reprehensión y castigo de los cuales se puede sacar importante provecho para el exercicio de las virtudes, y aborrecimiento de los vicios, que es medio cierto y seguro con que se consigue la vida eterna : colegido de historias divinas y humanas. Cuenca : Juan Masselin, 1594.

Virués, Cristóbal. El Monserrate, Madrid : Sancha, 1805 [1588].

Zorrilla, José. Leyendas. Ed. Salvador García Castañeda. Madrid : Cátedra, 2010 [1837-1883].

Zorrilla, José. Obras Completas. Barcelone : Sociedad de Crédito Intelectual, 1884.

\section{NOTES}

1. J. de Zorrilla, "La pasionaria. Cuento fantástico », première édition dans Cantos del trovador, 1840 (Zorrilla $1884: 359$ ). Toutes les traductions françaises sont de notre fait.

2. Cf. le compte-rendu de l'ouvrage de Jacob Grimm « Forêt de romances espagnols », X. Marmier, Revue de Paris 28 (1836) : «Il nous semble que ces deux traditions espagnole et allemande, mises l'une à côté de l'autre, peuvent servir à caractériser la poésie populaire des deux pays. Dans la première, le récit est simple et naturellement conçu. Il n'y a là ni fable ni apparitions merveilleuses ; c'est le griffon, c'est le diable, c'est le lion. Les romances espagnols sont en effet presque entièrement dépourvus de merveilleux, et il apparait à chaque page dans les anciennes ballades allemandes. On dirait que le ciel brumeux du Nord enfante naturellement les créations étranges, et que la limpide clarté du soleil d'Espagne dissipe ces images nébuleuses, ces figures ossianiques. » (p. 236)

3. Pour une biographie de José de Zorrilla, voir l'introduction de Salvador García Castañeda à l'édition de Cátedra des Leyendas, Madrid, 2010.

4. Le « romance " est un poème bref, composé d'octosyllabes et dont les vers pairs sont assonantiques. Le romantisme procède à une récupération du romancero comme partie intégrante de l'esprit national espagnol: en Espagne, cette récupération commence avec la publication en 1821 de Colecciones de romances antiguos o Romanceros, Valladolid, par Agustín Durán.

5. À propos de la métrique de Zorrilla, voir García Castañeda 39.

6. Dans son étude sur la formation de cette légende, R. Miquel y Planas signale comme probable origine la vie de Saint Jacques l'Ermite ou l'Ascète (VI ${ }^{\mathrm{e}}$ siècle) : quoique la fin soit différente car la jeune fille n'est pas retrouvée vivante, cette origine hagiographique permet d'expliquer l'existence d'une légende musulmane proche, celle du moine Barsisa, qui puiserait également 
dans Métaphraste, hagiographe byzantin.

Parmi les chroniques historiques faisant état de la légende, il cite Fray Pedro de Burgos, Libro de la Historia y Milagros de Nuestra Señora de Montserrat, Barcelone, 1514; P. Antonio Vicente Doménech, Flos Sanctorum o Historia General de los santos y varones ilustres en santidad del Principado de Cataluña, 1602; Francisco Diago Historia de los victoriosissimos antiguos Condes de Barcelona, 1603; P. Antonio Yepes, Crónica de la Orden de San Benito, 1609; Fray Gregorio de Argaiz, La Perla de Cataluña. Historia universal de Nuestra señora de Monserrate, Madrid: 1677; Pedro Serra y Postius, Epítome histórico, del portentoso Santuario y Real Monasterio de Nuestra Señora de Monserrate, tome premier, Barcelone, 1742. Joaquín de Entrambasaguas cite également une Historia de Fra Gari, ermita de Montserrat, d'Antonio Bulbena, 1822 (Entrambasaguas 111) : nous n'avons pas pu consulter cette œuvre et ne savons donc pas s'il s'agit d'un ouvrage historique ou littéraire.

7. La seconde édition a été publiée dans une collection entre 1771 et 1772 , à Valence : Impr. Agustín Laborda. Une troisième édition apparaît en 1772 à Murcie : Impr. De Francisco Benedito. L'édition de 1778, publiée à Madrid, chez l'imprimeur Manuel José Martín, est la première à citer l'imprimeur comme auteur de l'œuvre. On trouve une autre édition, sans date, publiée à Cordoue, chez Juan Rodríguez de la Torre, et qui indique aussi que l'auteur est Manuel José Martín et non pas Hilario Santos Alonso : cette édition, qui est consultable sur Google Books, est celle que nous avons utilisée mais nous indiquerons le véritable auteur de l'œuvre dans nos citations.

8. En 1850, Víctor Balaguer propose une réécriture en prose de la légende, moins fidèle à la tradition que Zorrilla, dans Monserrate. Su historia. Sus tradiciones. Sus alrededores, Barcelone : A. Brusi. Miquel y Planas cite également Luis Obiols, Garín. Leyenda histórica popular, Barcelone : Maucci, 1894. Pour la France, il cite Maurice Spronch, La légende de Jean Gari, Figaro illustré, 1891 et Marius André, Montserrat, roman féérique, 1896. Joaquín de Entrambasaguas ajoute El Ermitaño de Monserrate, roman historique de Torcuato Tárrago y Mateos, Madrid, 1861 ; Joan Garín o las montanyas de Monserrat, drame de Jaime Piquet y Piera, 1872; Nuestra Señora de Montserrat, de Julián Castellanos y Velasco, in Advocaciones de la Virgen, tome II, 1886; Garín, tradition catalane d'Apeles Mestres, Barcelone, 1898; Garín, opéra de Tomás Bretón de 1892 (Entrambasaguas 218).

9. Selon les versions, l'ermite se nomme Garí, Guarín ou Garín: seul Zorrilla transforme légèrement son nom en «Guarino ", sans doute pour espagnoliser un nom catalan.

10. Les citations où ne sont indiqués que les vers entre parenthèses appartiennent toutes à « $L a$ azucena silvestre ", de Zorrilla.

11. La vida de fray Juan Guarín possède une illustration représentant une bête à tête humaine. La version «de cordel » éditée par Hernando possède également une illustration représentant la scène de la chasse de la bête monstrueuse, voir fig. 2.

12. "A causa del calor, y frío le nació tanto vello, y cabellos, que parecía salvaje. " ("A cause de la chaleur et du froid, il lui poussa tant de poils et de cheveux, qu'il ressemblait à une bête sauvage. ») (Font 19)

13. Madrid: D. José M. Moreno, 1858. La mule étant un hybride de l'âne et de la jument, elle est méprisée comme tous les animaux bâtards. En outre, comme l'âne, elle peut être considérée comme symbole de la lascivité (Pastoureau 104-107).

14. Un dragon lui brûle les entrailles pour ses « saletés ", des couleuvres lui mordent la poitrine pour les caresses qu'elle a reçues; des crapauds lui arrachent les yeux pour avoir regardé des choses vaines et illicites; des chiens lui mordent les mains pour ne pas avoir fait l'aumône ( Discurso décimo, « De castidad », Villegas 111).

15. Curioso romance, del exemplar castigo que Dios nuestro Señor ha permitido en un Caballero, por haber levantado un falso testimonio a una Doncella honesta, llamada Dionisia Pérez Losada. [...], Madrid: Marés, 1847 : « quatre horribles démons / entrèrent dans la chambre / sous la forme de chiens affreux, / le saisirent rageusement, / et là en présence de tous / ils le traînent sur le sol, / en lançant d'horribles aboiements, / et à haute voix déclarent : / Voilà la justice, disent-ils, 
/ Que le Très-Haut nous ordonnent / d'exécuter sur cet homme / [...] Il ordonne donc que nous l'emportions / en enfer corps et âme. »

16. Contemplant la montagne, Guarino indique la corrélation entre la nature et Dieu : « Todo, todo de su Dios / el poder le manifiesta, / y él le conoce y le adora / en sus obras más pequeñas. » (v. 623-626) (" Tout, tout de son Dieu / lui manifeste le pouvoir, / et il le connaît et l'adore / dans ses plus petites créations. »)

17. Dans la tradition chrétienne, à la suite de la Genèse et de l'Apocalypse selon saint Jean, les reptiles (serpents, dragons, basilics, etc.) sont associés au démon, ce que révèlent les bestiaires et les livres d'exemples médiévaux.

18. «Recibióle la dama generosa, / mostrando [...] / que sin consuelo, triste y temerosa / había estado mientras dél ausente : / esto diciendo con tan dulce acento, / que por oírla se paraba el viento. » (« La dame le reçut aimablement, / lui indiquant qu'elle avait été inconsolable, triste / et apeurée pendant son absence : / elle prononça ces mots si délicatement, / que le vent s'arrêtait pour l'écouter. ») (Virués 22)

19. « La hija del conde, ofreciendo a las miradas del ermitaño su seno y sus hombros medio desnudos, con su ardiente mirada fija en el anacoreta, con ademanes voluptuosos y palabras que se filtraban en el alma de Juan Garín como un veneno sutil [...]. » (« La fille du comte, offrant aux regards de l'ermite son sein et ses épaules à demi-nus, son regard ardent fixé sur l'anachorète, avec des gestes voluptueux et des paroles qui s'insinuaient dans l'âme de Juan Garín comme un subtil venin [...] ) (Historia de la aparición 10)

20. «El tomillo oloroso, / la madreselva espesa, / la ancha amapola en su capullo aún presa, / el silvestre jacinto / que a la margen sonora / crece del arroyuelo [...] Nueva generación de mariposas / y de varios insectos zumbadores / ensayaban su vuelo en las hojosas / matas espesas de silvestres flores. / Los blancos conejuelos, / los alegres y libres cervatillos, / de su fuerza primera / iban ya haciendo alarde en la carrera. » (« Le thym parfumé, / le chèvrefeuille épais, / le large coquelicot encore prisonnier de son bourgeon, / la jacinthe sauvage / qui pousse sur la berge / sonore du ruisseau [...] Une nouvelle génération de papillons / et de divers insectes bourdonnants / tentent leur envol dans les fourrés / épais de fleurs sauvages. / Les petits lapins blancs, / les porte-muscs joyeux et libres, / faisaient déjà montre / de leur première force dans la course. ») (v. 2625-2630, 2650-2657)

21. "Purísima azucena » (« lys très pur ») (v. 2763) ; « pies purísimos » (de María) (" pieds très purs ») (v. 2920) ; « la Purísima Madre del Dios niño. » (« la Très Pure Mère de l'Enfant Jésus. ») (v. 3006)

22. Il est peu probable que Zorrilla ait eu connaissance de la symbolique de la fleur bleue développée par Novalis car, contrairement à Goethe, Schiller, les frères Grimm, les frères Schlegel et Hoffmann, cet écrivain ne fait pas partie des auteurs allemands connus par les romantiques espagnols (Hans Juretschke 1975).

23. «Carta de Baltasar Escobar », in El Monserrate, p. XXVI.

24. Dans «La corza blanca " (1863), l'héroïne, qui se transforme en biche toutes les nuits, illustre la vision médiévale de la femme qui associe sensualité et bestialité. Dans «Creed en Dios » (1862), la mère du héros, enceinte de lui, voit en songe un serpent grandir dans son sein, présage funeste du caractère diabolique de son fils. Dans « La ajorca de oro » (1861), un amoureux volant un bijou à la Vierge pour sa fiancée, meurt de peur en voyant s'animer les statues des saints ainsi que tous les démons représentés sous forme de reptiles présents dans la cathédrale.

25. On trouve aussi chez Bécquer des exemples d'animaux ambigus : dans «Creed en Dios » (1862), un mystérieux page apporte au noble sacrilège, un cheval noir, qui laisse penser à un présent diabolique, mais qui permet pourtant au chevalier de se repentir en lui faisant voir le Paradis et l'Enfer. 


\section{RÉSUMÉS}

L'écrivain espagnol José de Zorrilla, auteur de nombreuses légendes, à mi-chemin entre tradition religieuse espagnole et fantastique hoffmannien, place le monde naturel au centre de l'une d'elles, « La azucena silvestre » (1845). Traversée par une ligne de fracture manichéenne, la nature devient l'enjeu de la poétique de l'auteur, entre tradition et rénovation, entre merveilleux chrétien et ambiguïé fantastique.

\section{AUTEUR}

\section{JUSTINE PÉDEFLOUS}

Justine Pédeflous est professeur agrégé d'espagnol et prépare à Paris - Sorbonne une thèse portant sur la dimension morale de la littérature fantastique espagnole du XIX ${ }^{\mathrm{e}}$ siècle. Elle s'intéresse particulièrement à la problématique de l'articulation entre exemplarité et esthétique ainsi qu'à la place de la religion dans la littérature espagnole du XIX ${ }^{\mathrm{e}}$ siècle. Elle a déjà publié plusieurs articles sur le genre fantastique mais également sur d'autres formes littéraires nonréalistes, comme les romances de ciegos religieux, la science-fiction, le conte spiritiste ou encore le conte de fées. Elle a également publié une co-traduction aux Belles-Lettres d'une biographie de l'humaniste espagnol Juan Luis Vives. 